\title{
Motive und Rationalität des Protektionismus. Einige einfache spieltheoretische Überlegungen
}

\author{
Jürgen Jerger
}

Online publiziert: 14 . Oktober 2020

(C) Der/die Autor(en) 2020

Zusammenfassung Dieser Beitrag nutzt einen einfachen spieltheoretischen Rahmen, um die protektionistischen Tendenzen der jüngeren Vergangenheit als Ergebnis der Interaktion von politischen Entscheidungsträgern in zwei Ländern zu verstehen. Die (in diesem Rahmen: binäre) Politikentscheidung zwischen Protektionismus und Freihandel hängt natürlich von den erwarteten wahrgenommenen Ergebnissen dieser Politikoptionen ab. Die Wahrnehmung dieser Ergebnisse steht dabei im Fokus dieses Papiers. Es werden die Bedingungen untersucht, unter denen Freihandel das Ergebnis der beiden Politikentscheidungen bleibt bzw. wann sich dieses Ergebnis in ein protektionistisches Gleichgewicht ändert.

Schlüsselwörter Protektionismus · Handelspolitik · Spieltheoretische Analyse

\section{On the rationality of protectionism. Some simple game-theoretic considerations}

\begin{abstract}
This paper employs a simple analytical framework in order to understand the recent protectionist developments as a result of the interaction of policymakers in two countries. Quite naturally, the (in this framework: dual) decision between free trade and protectionism criticalls depends on the expected perceived outcomes of the options available to the political decision-makers. The perception of these outcomes is in the focus of the paper. We look at the conditions under which free trade remains the outcome of the joint policy decisions and when this outcome is changed to a protectionist equilibrium.
\end{abstract}

Keywords Protectionism · Trade policy $\cdot$ Game theoretic analysis

J. Jerger $(\square)$

Dept. of Economics, University of Regensburg, 93040 Regensburg, Deutschland

E-Mail: j.jerger@ur.de 


\section{Einleitung}

Die Jahrestagung 2020 des Ausschusses für Wirtschaftspolitik stellte die $R \boldsymbol{e}$ naissance der Markteingriffe in den Mittelpunkt. Und in der Tat gibt es viele Politikbereiche, in denen Markteingriffe wieder eine stärkere Rolle spielen. ${ }^{1}$ Dazu gehört seit der Wirtschafts- und Finanzkrise 2008/09 vor gut einem Jahrzehnt auch der Außenhandel. Waren die gut sechs Jahrzehnte nach dem zweiten Weltkrieg durch eine zwar nicht gleichmäßige, so doch in der Richtung unveränderte Liberalisierung des weltweiten Außenhandels geprägt, so hat sich dies seit gut einer Dekade verändert. Offen protektionistische Tendenzen sind (wieder) relevant geworden - bis hin zur deutlichen Schwächung multilateraler Foren, im Kontext der Handelspolitik insb. der Welthandelsorganisation. Die von US-Präsident Donald Trump ausgegebene Parole America first! ist nur die vermutlich kürzeste Art und Weise, expliziter (wirtschafts-) politischer Kooperation eine Absage zu erteilen. Äußerungen in diese Richtung beschränken sich aber in jüngerer Zeit weder auf die USA noch auf das Gebiet der Handelspolitik.

Dabei ist das Denken in nationalen Egoismen keineswegs neu, auch nicht im Kontext handelspolitischer Fragestellungen. Ganz im Gegenteil geht die politökonomische Analyse von Handelspolitik ausdrücklich davon aus, dass handelspolitische Entscheidungen egoistisch getroffen werden, d. h. vor dem Hintergrund von Bewertungen auf nationaler Ebene bzw. auf der Ebene von politischen Akteuren, die ihren Eigennutz verfolgen (Baldwin 1989; Rodrik 1995; Krueger 1996).

Auch an konkreten Mechanismen, die Protektionismus aus nationaler bzw. individueller Perspektive wünschenswert erscheinen lassen können - und damit politökonomisch erklärbar machen -, besteht keinerlei Mangel.

So kann entlang des altbekannten Optimalzollarguments (Bhagwati und Ramaswami 1992) eine große Volkswirtschaft durch einen Importzoll den Weltmarktpreis für Importgüter senken, wenn die Verteuerung des Konsumentenpreises die weltweite Nachfrage hinreichend stark senkt. Diese mögliche Verbesserung der terms of trade kann - muss aber nicht - auch zu einer gesamtwirtschaftlichen Wohlfahrtsverbesserung führen.

Ein zweites - und in der öffentlichen Diskussion sehr viel prominenteres Erklärungsmuster für Protektionismus sind die mit Freihandel verbundenen Verteilungswirkungen. Bereits das Heckscher-Ohlin-Modell impliziert ja, dass der in einer Volkswirtschaft relativ zum Rest der Welt eher knappe Produktionsfaktor durch Außenhandel verliert (Stolper-Samuelson-Theorem), auch wenn damit positive Wohlfahrtseffekte auf gesamtwirtschaftlicher Ebene verbunden sind. Wenn eine hinreichende Kompensation der Verlierer gesellschaftlich nicht organisiert werden kann, werden die sich also aus wohlverstandenem Eigeninteresse für Protektionis-

\footnotetext{
1 Die sowohl quantitativ als auch qualitativ massiven Staatseingriffe im Zuge der Corona-Krise haben diese Entwicklung noch verstärkt. Schon nur die Festlegung des Themas weit vor dieser Krise zeigt jedoch, dass die Tendenz dazu auch zuvor deutlich und wirtschaftspolitisch relevant war.

2 Vgl. Cernat und Mustilli (2018) für eine Diskussion des Einsatzes des 2006 aufgelegten European Globalisation Adjustment Funds. Südekum (2017) weist auf die Bedeutung der regionalen Konzentration schrumpfender Branchen für die Ausgestaltung von Kompensationsschemata hin.
} 
mus einsetzen. ${ }^{2}$ In jüngerer Zeit sind es empirische Arbeiten z. B. von Autor et al. (2013), Autor et al. (2017) und Dauth et al. (2017), in denen die Verlierer - insb. Arbeiter in importkonkurrierenden Sektoren - identifiziert werden. van Paridon (2018) beschäftigt sich ausführlich mit den möglichen negativen Rückwirkungen der Globalisierung und dem dadurch forcierten Strukturwandel auf das soziale Gefüge und dessen Stabilität.

Natürlich hängen die politischen Entscheidungen auch davon ab, wie gut die jeweiligen Interessen der betroffenen Gruppen organisiert werden können (Olson 1965). In dem Maße, in dem tatsächliche oder vermeintliche Verlierer offener Grenzen sich im politischen Prozess besser durchsetzen können bzw. eine Kompensation dieser Verluste nicht gelingt, wird eine im Ergebnis protektionistische Politik wahrscheinlicher.

Der Rest dieses Beitrags ist wie folgt aufgebaut: Im folgenden Abschn. 2 wird kurz auf das seit neuerem verbreitete Schlagwort der Deglobalisierung eingegangen. Abschn. 3 präsentiert dann unterschiedliche Szenarien der strategischen handelspolitischen Interaktion zweier Länder. Die Szenarien unterscheiden sich hinsichtlich der Auszahlungen bzw. wahrgenommenen Auszahlungen in den möglichen Konstellationen, die sich aus den handelspolitischen Entscheidungen ergeben können. Ein kurzes Fazit wird in Abschn. 4 gezogen.

\section{Deglobalisierung?}

Seit bereits einiger Zeit gibt es eine Literatur, die sich mit dem empirisch noch bestreitbaren und daher auch umstrittenen Phänomen der Deglobalisierung, d.h. einer wieder weniger eng werdenden internationalen (Handels-) Verflechtung, beschäftigt. Schon 2015 wurde das etwas mildere Schlagwort der „Slowbalisation“ von dem sich selbst als „trendwatcher“ bezeichnenden Publizisten Adjiedi Bakas geprägt.

Abb. 1 zeigt die Entwicklung der Handelsintensitäten für Deutschland, die USA und weltweit (Datenquelle: https://data.worldbank.org/indicator/ne.trd.gnfs.zs). Diese ist jeweils gemessen als prozentualer Anteil der Summe von Importen und Exporten am Bruttoinlandsprodukt. Weltweit hat sich in den knapp vier Jahrzehnten

Abb. 1 Handelsintensitäten weltweit, in den USA und in Deutschland (Worldbank)

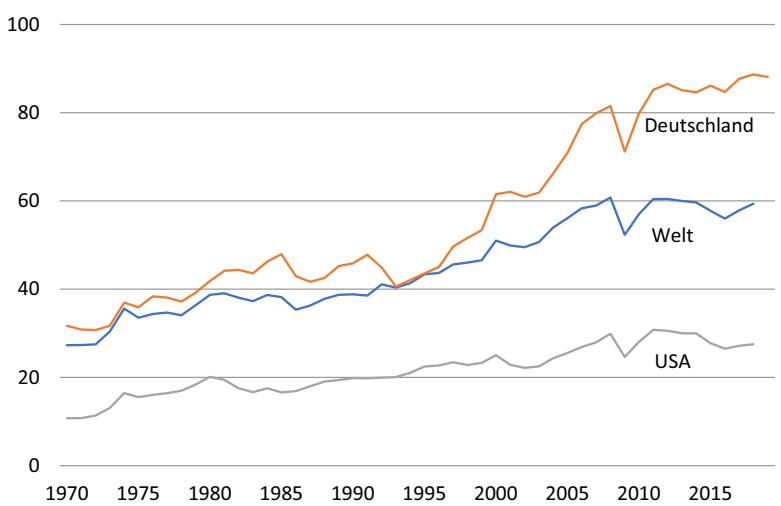


bis zur Wirtschafts- und Finanzkrise 2008/09 die Handelsintensität fast verdreifacht. Der deutliche Einbruch während der Krise wurde dann zwar wieder aufgeholt, die zuvor beobachtbare Dynamik hat jedoch eindeutig nachgelassen. In Deutschland ist die Handelsintensität demgegenüber am Datenrand zwar höher als vor Beginn der Wirtschafts- und Finanzkrise, aber auch hier kam das rasante Wachstum seit Beginn der 1990er Jahre fast vollständig zum Erliegen. Die aktuelle Covid-Pandemie wird aufgrund des zeitweisen Ausfalls wichtiger Transportwege nochmals einen deutlichen Rückgang der Handelsintensität mit sich bringen (WTO 2020).

Der massive Anstieg der Handelsintensität bis vor etwa einem Jahrzehnt wurde vielfach als ein mehr oder weniger naturgesetzlich ablaufender Prozess der zunehmenden Globalisierung, insb. getrieben durch sinkende Transportkosten und die Effizienzvorteile einer immer tiefergehenden internationalen Arbeitsteilung. Eine ganz wesentliche Voraussetzung für diese Entwicklung war jedoch deren Ermöglichung durch die Politik. Innerhalb der EU wurden Handelsbarrieren durch die Schaffung des Binnenmarktes praktisch völlig eliminiert, weltweit waren es insb. die Entscheidungen von China, Indien und den Nachfolgestaaten der ehemaligen Sowjetunion für die recht weitgehende Öffnung ihrer Grenzen. Diese freihandelsorientierte politische Dynamik hat jedoch zuletzt deutlich nachgelassen. Der (dann später frühzeitig zurückgetretene) Generaldirektor der Welthandelsorganisation, Roberto Azevêdo, beschrieb dies wie folgt: „Mounting trade tensions, an increase in trade-restrictive measures and continuing economic uncertainty created real challenges for world trade in 2018.“ (WTO 2019, S. 8). Die Corona-Pandemie scheint die protektionistischen Instinkte in der Politik noch mehr zu befeuern. So hat der Bundesgesundheitsminister Jens Spahn in einer Pressekonferenz am 2. März 2020 erklärt, ,,..., dass wir über das richtige Maß an Globalisierung werden reden müssen.“ Eine weitere politische Tendenz, die dem Protektionismus Vorschub leistet, ist der Einsatz von Handelssanktionen, insb. gegenüber Russland im Gefolge der Annexion der Krim und gegenüber dem Iran.

Ein gemischtes empirisches Bild ergibt sich aus der Entwicklung wichtiger regionaler Freihandelsabkommen. Während die anvisierte tiefere Integration zwischen den USA und wichtigen asiatischen Staaten (das sog. Trans-Pacific Partnership, TTP) einerseits und der EU (Transatlantic Trade and Investment Partnership, TTIP) andererseits nicht zustande gekommen sind, konnten z. B. die Abkommen zwischen der EU und Kanada sowie Japan geschlossen werden.

Aus all diesen Beobachtungen folgt, dass jedenfalls die Intensivierung der internationalen Verflechtung bei weitem nicht mehr so dynamisch ist wie in der Vergangenheit. Inwieweit größere Sorgen bzgl. einer politisch gewollten und durchgesetzten Entflechtung berechtigt sind, wird erst die Zukunft weisen. van Bergeijk $(2018,2019)$ sieht jedenfalls bereits eine hinreichende Grundlage dafür, von einer zweiten Deglobalisierungswelle (nach den 1930 Jahren) zu sprechen. Allerdings ist dieser Befund noch nicht allgemein konsensfähig, siehe bspw. Bordo (2017). Die in Abb. 1 gezeigten durchaus unterschiedlichen Entwicklung für Deutschland und die USA machen deutlich, dass die Faktenlage hier noch nicht wirklich eindeutig ist.

Es erscheint aber angebracht, die wirtschaftshistorische Warnung eines massiven Handelskollaps von Charles P. Kindleberger in Erinnerung zu rufen. Abb. 2 - die berühmte Kindleberger-Spirale - zeigt den Rückgang des Welthandelsvolumes um 
Abb. 2 Die Kindleberger-Spirale (Kindleberger 1973, S. 172)

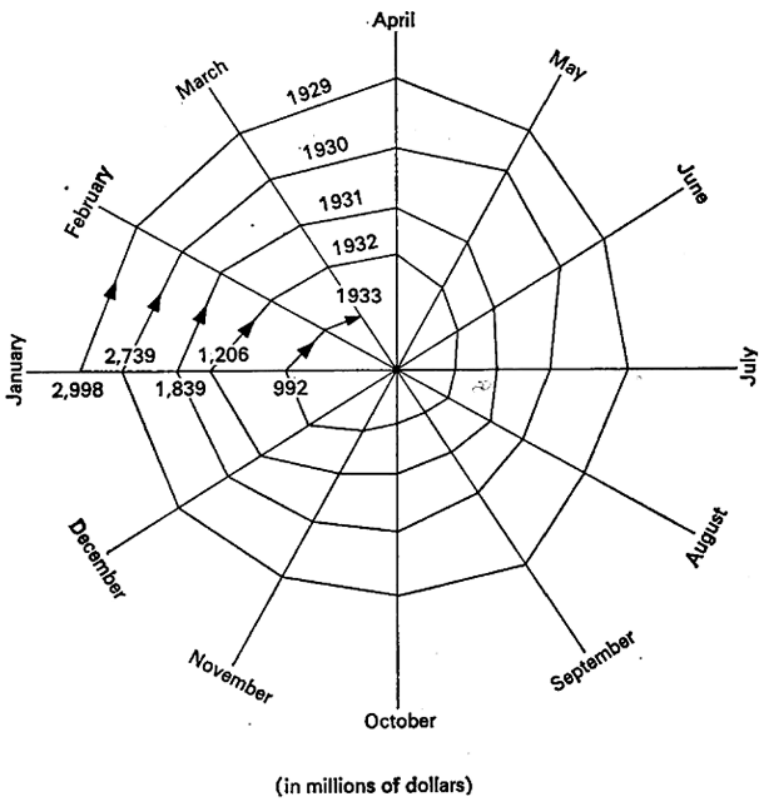

Figure 8. The contracting spiral of world trade, January 1929 to March 1933: total imports of seventy-five countries (monthly values in terms of old U.S. gold dollars (millions) ).

mehr als zwei Drittel in dem kurzen Zeitraum zwischen 1929 und 1933. Diese Entwicklung war direktes Resultat von Politikentscheidungen, insb. der Verabschiedung des Smoot-Hawley Act im Jahr 1930. Es ist vielleicht auch für die aktuelle Situation ganz instruktiv, dass sich damals wichtige Wirtschaftsvertreter - u. a. Henry Ford und das akademische Who Is Who der amerikanischen Wirtschaftswissenschaften gegen dieses Gesetz ausgesprochen haben und dass auch zahlreiche ausländische Regierungen offiziell Protest einlegten. Die - natürlich trügerische - Hoffnung auf eine Verbesserung der Lage der amerikanischen Arbeiter durch Handelsrestriktionen führte aber bekanntermaßen zur Verabschiedung des Gesetzes. Die erwartbare Reaktion des Auslandes auf die amerikanischen Importzölle war selbstredend die Einführung von Retorsionszöllen. Es gibt heutzutage leider wenig Anlass zu der Hoffnung, dass dieser für Handel und Wohlstand mit negativen Folgen behaftete Mechanismus nicht wieder zum Tragen kommen könnte.

\section{Einige theoretische Überlegungen (zu strategischen handelspolitischen Spielen)}

\subsection{Die Idee}

Handelspolitische Entscheidungen können als Ergebnis der strategische Interaktion der beteiligten Länder, die jeweils ihre eigene Interessenslage im Blick haben, 
verstanden werden. Diese Denkweise ist spätestens seit den Beiträgen von Bagwell und Staiger $(1999,2000)$ in der Literatur fest verankert. Die nachfolgend genutzte Stilisierung dieser Analyse durch die Annahme einer dichotomen Wahl zwischen „Freihandel“ und „Protektionismus“ anstelle einer stetig zu variierenden Politikvariable wie den Zollsatz wird bspw. in dem Lehrbuch von Krugman et al. (2018, S. 289) seit langem benutzt. Konkret wird dort die Sinnhaftigkeit eines multilateralen Verhandlungsrahmens wie der World Trade Organization bzw. des GATT als Weg aus einem möglicherweise vorhandenen Gefangenendilemma illustriert; dieses Szenario wird nachfolgend in Abschn. 3.5 behandelt.

Grundsätzlich sind jedoch die Konsequenzen der strategischen Interaktion zweier Länder, die eine entweder liberale oder protektionistische Handelspolitik betreiben können, durchaus nicht unstrittig. Folgt man der traditionellen Außenhandelstheorie und geht insb. von der Annahme nicht vermachteter Märkte aus, so ergeben sich eindeutig negative Konsequenzen eines auch unilateralen Protektionismus. Zwar ergeben sich durch die Berücksichtigung unvollständiger Konkurrenz mögliche Argumente zugunsten des Protektionimus aus zumindest nationaler Perspektive, in einer frühen Bewertung dieser Implikationen hat aber der Pionier dieser Literatur, Paul R. Krugman, die Frage, ob damit Freihandel passé sei, klar verneint (Krugman 1987). Daher wird dieses Szenario als „ehrliches Szenario“ bezeichnet.

Dieses und die weiteren Szenarien lassen sich jeweils begründen durch unterschiedliche Bewertungen der Konsequenzen von Protektion bzw. Freihandel. Konkret werden die folgenden Szenarien behandelt, zu denen sich jeweils eine politische Rationalität der entsprechenden Bewertungen angeben lässt - und die zu jeweils sehr unterschiedlichen Ergebnissen führen:

- Das ehrliche Szenario

- Das fast ehrliche Szenario

- Mildes GATT-think

- GATT-think

- Harter Nationalismus

Aufgrund der oben bereits eingeführten dichotomen Politikwahl und der Annahme, dass die strategische Interaktion zwischen nur zwei Ländern - nachfolgend Land A und Land B genannt - untersucht wird, gibt es jeweils vier mögliche Ergebnisse. Dieser Modellrahmen ist damit der denkbar einfachste, in dem die Konsequenzen der strategischen handelspolitischen Interaktion untersucht werden können. Es wird dabei stets unterstellt, dass die politischen Akteure rational handeln, d. h. ihre eigene Zielfunktion maximieren. Technisch gesprochen werden daher die Nash-Gleichgewichte der handelspolitischen Entscheidungen identifiziert.

\subsection{Das ehrliche Szenario}

Ungehinderter Freihandel, d.h. die Absenz von Protektionimus, kann als die einmütige Empfehlung der wissenschaftlichen Beschäftigung mit Handelsrestriktionen bezeichnet werden, wenn die Maximierung der gesamtgesellschaftlichen Wohlfahrt das Ziel der politischen Entscheidungsträger ist. In theoretischen Modelle ohne Marktmacht trifft dies ohne jede Einschränkung zu, aber auch empirische Analy- 
Tab. 1 Das ehrliche Szenario

\begin{tabular}{llcc}
\hline & & Land B & \\
& & Freihandel & Protektionismus \\
\hline Land & Freihandel & $(\underline{10}, \underline{10})$ & $(\underline{9}, 8)$ \\
A & Protektionismus & $(8, \underline{9})$ & $(7,7)$ \\
\hline
\end{tabular}

sen kommen praktisch ausnahmslos zu diesem Ergebnis. So wird in der Profession beispielsweise auch einmütig davon ausgegangen, dass die durch den Brexit geschaffenen zusätzlichen Handelsbarrieren sowohl das Vereinigte Königreich als auch wenngleich schwächer ausgeprägt - den verbleibenden EU-27-Ländern wirtschaftlich schaden (Sachverständigenrat 2018, S. 28). Es ist dabei fast schon ironisch, dass einer der wenigen Befürworter des Brexit aus den Reihen der Wissenschaft dessen vorgeblich segensreichen Wirkungen im Wesentlichen darauf zurückführt, dass das Vereinigte Königreich außerhalb der EU - d.h. mit dem Rest der Welt - liberalere Handelsabkommen schließen kann (Minford 2017). Damit haben Gegner und Befürworter des Brexit ihre Gemeinsamkeit in der Unterstützung einer möglichst liberalen Handelspolitik. Der professionelle Streit um die ökonomischen Wirkungen des Brexit geht also letztlich um eine Einschätzung wie schnell und wie umfassend das Vereinigte Königreich mit dem Rest der Welt wirklich weitgehende Freihandelsabkommen abzuschließen in der Lage ist. Dass man in dieser Fage weniger optimistisch als Patrick Minford ist, erscheint dabei als durchaus realistisch.

Dieses Szenario kann durch die - selbstredend stilisierte - Auszahlungsstruktur in Tab. 1 abgebildet werden. Konkret wird hierbei angenommen, dass

1. Freihandel für beide Länder am besten ist;

2. unilateraler Protektionismus beiden schadet;

3. und ein Land durch eine protektionistische Politik mehr verliert als dasjenige, das bei Freihandel bleibt.

Unter diesen Annahmen ist das Ergebnis der strategischen Interaktion fast schon trivial. Freihandel ist für beide Länder die dominante Strategie, das eindeutige NashGleichgewicht ist zugleich Pareto-optimal. Wenn also die in Tab. 1 unterstellte Struktur der Auszahlungen eine zutreffende Beschreibung der Realität ist und wenn die politischen Entscheidungsträger diese so wahrnehmen, dann gibt es keinerlei Problem. Eine „MyCountry first!“-Politik würde in diesem Fall einer handelspolitischen Liberalisierung nicht im Weg stehen. Schon nur die Tatsache, dass handelspolitische Auseinandersetzungen immer relevant waren und auch weiterhin sind, macht es aber notwendig, weitere Szenarien in Erwägung zu ziehen. Denn ganz offensichtlich ist die handelspolitische Realität mit dem ,ehrlichen Szenario“ nicht abzubilden, selbst wenn die theoretischen und empirischen Grundlagen der Auszahlungsstruktur in Tab. 1 sehr solide sind.

\subsection{Das fast ehrliche Szenario}

Das erste dieser alternativen Szenarien sei als „fast ehrlich“ bezeichnet und unterscheidet sich von dem ehrlichen Szenario nur dadurch, dass nun anstelle der o.g. 
Tab. 2 Das fast ehrliche Szenario

\begin{tabular}{llcc}
\hline & & Land B & \\
& & Freihandel & Protektionismus \\
\hline Land & Freihandel & $(\underline{10}, \underline{10})$ & $(\underline{8}, 9)$ \\
A & Protektionismus & $(9, \underline{8})$ & $(7,7)$ \\
\hline
\end{tabular}

Annahme 3. angenommen wird, dass ein Land durch eine protektionistische Politik sich selbst weniger schadet als dem jeweiligen Handelspartner. Die Wahrnehmung einer solchen Auszahlungsstruktur könnte beispielsweise dadurch begründet werden, dass die Lobby des importkonkurrierenden Sektors eine erfolgreiche Arbeit betreibt. Die Annahmen 1. und 2. des Szenarios von Abschn. 3.2 werden beibehalten. Dieses Szenario kann durch die Auszahlungsstruktur in Tab. 2 erfasst werden.

Unter diesen Annahmen bleibt Freihandel die dominante Strategie für beide Länder, es ändert sich gegenüber dem ehrlichen Szenario nichts am Ergebnis, das auch nach wie vor die Eigenschaft der Pareto-Optimalität aufweist. Selbst wenn also die in den traditionellen Außenhandelsmodellen enthaltene Implikation der stärkeren Selbst- relativ zur Fremdschädigung durch eine protektionistische Politik nicht greift bzw. nicht so wahrgenommen wird, wäre dies kein Anlass zur Besorgnis in Kategorien des handelspolitischen Ergebnisses.

\subsection{Mildes GATT-think}

Paul Krugman (1991, S. 25) führte die drei folgenden normativen Positionen als Charakterisierung der an den GATT-Handelsrunden beteiligten Länder bzw. Akteure auf:

1. Exporte sind gut.

2. Importe sind schlecht.

3. Ceteris paribus ist ein gleich hoher Anstieg von Importen und Exporten gut.

Nur vor diesem Hintergrund dieser Bewertungen - so Krugman - ist verständlich, warum es zum einen ein multilaterales Verhandlungsforum wie das GATT bzw. die WTO überhaupt braucht und dieses dann zu wechselseitig vorteilhaften Ergebnissen kommen kann. Mit diesen Bewertungen konsistent sind die Annahmen, dass

1. bilateraler Freihandel für beide Länder besser als bilateraler Protektionismus ist;

2. unilateraler Protektionismus dem jeweils anderen Land schadet, dem protektionistischen Land selbst jedoch nützt;

3. unilaterale Protektion ein Nullsummen-Spiel in dem Sinn ist, dass die Gewinne des protektionistischen Landes den Verlusten des anderen Landes, das bei Freihandel bleibt, entsprechen.

Die Auszahlungsmatrix in Tab. 3 spiegelt diese Annahmen wieder:

„Mild“ ist an diesem Szenario die dritte Annahme, dass eine unilaterale Protektion die Freihhandelsgewinne nur umverteilt, diese im Aggregat über beide Länder jedoch nicht geschmälert werden. 
Tab. 3 Mildes GATT-think Szenario

Land B

Freihandel Protektionismus

\begin{tabular}{llcc}
\hline Land & Freihandel & $(10,10)$ & $(\underline{9}, \underline{11})$ \\
$\mathrm{A}$ & Protektionismus & $(\underline{11}, \underline{9})$ & $(7,7)$ \\
\hline
\end{tabular}

In dieser Situation ist bilateraler Freihandel kein politökonomisches Gleichgewicht der strategischen Interaktion zwischen den beiden Ländern mehr. Vielmehr ergeben sich multiple, sich wechselseitig ausschließende Nash-Gleichgewichte. Ohne weitere Annahmen ist eine Selektion zwischen diesen Gleichgewichten nicht möglich. Ein multilateraler Verhandlungsrahmen kann hier möglicherweise die Koordination auf die Freihandelslösung ermöglichen; allerdings wird diese ggf. wenig stabil sein, da beide Länder einen Anreiz zur unilateralen Abweichung haben. Die Pareto-inferiore Situation eines bilateralen Protektionismus könnte jedoch als Drohpunkt für die Durchsetzung der Freihandelslösung dienen.

\subsection{GATT-think}

Ebenfalls konsistent mit der im letzten Abschnitt zitierten Charakterisierung des GATT-think von Krugman (1991) ist die Möglichkeit einer auch bei unilateralem Protektionismus deutlichen Wohlfahrtssenkung in dem Land, das bei Freihandel bleibt. Das protektionistische Land kann sich also gegenüber der Freihandelssituation verbessern auf Kosten starker Verluste desjenigen Landes, das bei einer liberalen Handelspolitik bleibt - wiederum ggf. nur in der Wahrnehmung der politischen Entscheidungsträger in den beiden Ländern.

Diese Annahmen werden durch die Auszahlungsmatrix in Tab. 4 reflektiert.

In diesem Fall liefert die strategische Interaktion zwischen den beiden Ländern wieder ein eindeutiges Nash-Gleichgewicht. Protektionismus wird nun zur dominanten Strategie. Damit besteht hier ein klassisches Gefangenendilemma - die individuell optimale Politikentscheidung führt zu einem kollektiv ineffizienten Ergebnis. Genau diese Struktur hat auch das in dem Lehrbuch von Krugman et al. (2018) behandelte Szenario. Ein multilateraler Verhandlungsrahmen kann hier durch wechselseitige Verpflichtungen zur Liberalisierung einen Weg aus dem Gefangenendilemma bieten, d.h. eine Koordination auf die Pareto-optimale Freihandelslösung. Wie immer im Gefangenendilemma besteht zwar ein unilateraler Anreiz zur Abweichung, die Liberalisierungserfolge im Rahmen des GATT bzw. der WTO und auch innerhalb großer regionaler Handelsbündnisse wie der Europäischen Union machen aber klar, dass offene Grenzen auch ein empirisch recht stabiles Ergebnis sein können.

Tab. 4 Das GATT-think Szenario

Land B

Freihandel Protektionismus

\begin{tabular}{llcc}
\hline Land & Freihandel & $(10,10)$ & $(6, \underline{11})$ \\
$\mathrm{A}$ & Protektionismus & $(\underline{11}, 6)$ & $(\underline{7}, \underline{7})$ \\
\hline
\end{tabular}


Abb. 3 Der US-amerikanische Präsident zu Handelskriegen (Twitter)
Donald J. Trump

@realDonaldTrump

When a country (USA) is losing many billions of dollars on trade with virtually every country it does business with, trade wars are good, and easy to win. Example, when we are down $\$ 100$ billion with a certain country and they get cute, don't trade anymore-we win big. It's easy! 10:50 AM - Mar 2, 2018

11.7K $\bigcirc 7,944$ people are talking about this

\subsection{Harter Nationalismus}

Die handelspolitischen Auseinandersetzungen in der jüngeren Vergangenheit deuten darauf hin, dass die vorgenannten Szenarien jedenfalls in einigen Situation bzw. aus der Sicht einiger politischer Akteure noch ,zu optimistisch“ sind, was die Durchsetzbarkeit der Freihandelslösung angeht. Protektionismus bzw. das dadurch implizierte Ergebnis kann - entgegen aller theoretischer Argumente und auch empirischer Evidenz - durchaus als der Freihandelslösung überlegen wahrgenommen werden. Diese Wahrnehmung ist keineswegs neu - und wurde von Kurt Tucholsky bereits im Jahr 1931 in seinem kurzen Abriss der Nationalökonomie wie folgt auf die Schippe genommen: „Weil billiger Weizen ungesund und lange nicht so bekömmlich ist wie teurer Roggen, haben wir den Schutzzoll, der den Zoll schützt sowie auch die deutsche Landwirtschaft.“ Insbesondere einige Äußerungen von Donald Trump auf Twitter gehen jedoch in genau diese Richtung - und wirken zumindest für einen Leser mit basalen Kenntnissen der Außenhandelstheorie kaum weniger satirisch. Beispielhaft dafür sei sein Tweet vom 2. März 2018 in Abb. 3 genannt. Offensichtlich verwechselt Trump ein Handelsbilanzdefizit mit einem Wohlfahrtsverlust und springt von diesem Mißverständnis zu der Folgerung, dass sein Land gewinnen könne, wenn dieser Handel wegfiele.

Die dieser Position zugrundeliegende Wahrnehmung sei hier bezeichnet als „harter Nationalismus“. Denn hier hat der Protektionismus einen Eigenwert, z. B. indem er dazu führt, dass die aus dem Freihandel resultierenden vermeintlichen Verluste vermieden werden. Wie gerechtfertigt oder unsinnig diese Position dann sein mag, steht auf einem anderen Blatt. Dies lässt sich durch die Auszahlungsstruktur in Tab. 5 erfassen.

Wie schon im GATT-think Szenario ist hier Protektionismus die dominante Strategie. Allerdings gibt es hier keinerlei Möglichkeit mehr, eine Freihandelslösung zu implementieren, einfach weil die wechselseitige handelspolitische Abschottung von beiden Ländern als das jeweils beste Ergebnis wahrgenommen wird. In einem solchen Szenario verliert auch die multilaterale Verhandlungsplattform der WTO ihre Daseinsberechtigung, da es ja keine bessere Verhandlungslösung gibt, die erzielt werden könnte. Insoweit ist die von Donald Trump sehr weitgehend betriebene Politik der Schwächung der WTO mit der Wahrnehmung einer Auszahlungsstruktur des handelspolitischen Spiels gemäß Tab. 5 konsistent. 
Tab. 5 Harter Nationalismus

\begin{tabular}{llcc} 
& & & Land B \\
& & Freihandel & Protektionismus \\
\hline Land & Freihandel & $(10,10)$ & $(9, \underline{11})$ \\
A & Protektionismus & $(\underline{11}, 9)$ & $(\underline{11}, \underline{11})$ \\
\hline
\end{tabular}

Das Szenario kann und muss als Warnung verstanden werden. Ein lang anhaltender und dramatischer Einbruch der Handelsströme mit all seinen negativen Konsequenzen für die Wohlfahrt war ein wesentliches Merkmal der Großen Depression in den 1930er Jahren. Dieser Einbruch war ein Resultat bewusster Politikentscheidungen, die letztlich auf der Überzeugung basierten, durch eine stärkere Abschottung gegenüber den Handelspartnern der Wirtschaft des jeweils eigenen Landes helfen zu können. Dass dies ein Trugschluss war und ist, kann als Konsensposition innerhalb der ökonomischen Zunft bezeichnet werden. Es ist ein theoretisch wie empirisch belastbares Faktum, dass die Auszahlungen bei wechselseitigem Freihandel (also oben links in den jeweiligen Tabellen) größer sind als bei wechselseitigem Protektionismus (unten rechts). Anders gesagt: Eine Handelspolitik, die sich auf die Auszahlungsstruktur von Tab. 5 stützt, basiert letztlich auf fake news. Die Wissenschaft bzw. wissenschaftliche Politik(er)beratung hatte hier lange Zeit insoweit eine einfache Aufgabe als die Wünschbarkeit von Freihandel recht wenig in Zweifel gezogen wurde. Und es ist letzlich auch egal, ob das Freihandelsergebnis auf den (fast) ehrlichen Szenarien der Tab. 1 bzw. 2 beruht oder auf der durch eine multilaterale Vereinbarung gestützte Lösung eines Gefangenendilemmas, wie in Tab. 4. Wenn sich die Wahrnehmung der Welt jedoch in Richtung Nationalismus verändert, dann ist es Aufgabe der Wissenschaft, die Auszahlungsstruktur in Tab. 5 als das zu bezeichnen was sie ist - eben schlicht unzutreffend.

\section{Fazit}

Das stetige Wachstum der Handelsintensität in den Jahrzehnten nach dem zweiten Weltkrieg ist vor nunmehr etwa einem Jahrzehnt deutlich eingebrochen. Seither entspricht das Wachstum der internationalen Handelsströme in etwa dem Wachstum der weltweiten Wertschöpfung. Jedenfalls teilweise hat diese Entwicklung zu tun mit protektionistischen Tendenzen, die bereits im Gefolge der Wirtschafts- und Finanzkrise 2008/09 spürbar waren, in letzter Zeit - und nicht zuletzt durch die Positionen und Entscheidungen des US-amerikanischen Präsidenten Donald Trump - aber weiter an Bedeutung gewonnen haben.

Es wird in diesem Papier anhand des in der Literatur gut eingeführten Modells der strategischen Interaktion handelspolitischer Entscheidungsträger gezeigt, dass Protektionismus entweder - und bekanntermaßen - als Pareto-inferiores Ergebnis eines Gefangenendilemmas verstanden werden kann oder aber auch aus einer Wahrnehmung der Überlegenheit protektionistischer Politik per se. Letzteres ist politökonomisch innerhalb eines Landes durch ein hohes Gewicht von Globalisierungsverlierern im politischen Entscheidungsprozess durchaus rationalisierbar. Allerdings 
wäre dies für die gesamtwirtschaftliche Ebene fatal. Die jüngsten handelspolitischen Entwicklungen geben Anlass zu der Forderung, dieses Wissen wieder offensiver in der Politik zu verankern, um die - nicht nur ökonomisch fatalen - handelspolitischen Fehler der 1930er Jahre nicht zu wiederholen.

Funding Open Access funding enabled and organized by Projekt DEAL.

Open Access Dieser Artikel wird unter der Creative Commons Namensnennung 4.0 International Lizenz veröffentlicht, welche die Nutzung, Vervielfältigung, Bearbeitung, Verbreitung und Wiedergabe in jeglichem Medium und Format erlaubt, sofern Sie den/die ursprünglichen Autor(en) und die Quelle ordnungsgemäß nennen, einen Link zur Creative Commons Lizenz beifügen und angeben, ob Änderungen vorgenommen wurden.

Die in diesem Artikel enthaltenen Bilder und sonstiges Drittmaterial unterliegen ebenfalls der genannten Creative Commons Lizenz, sofern sich aus der Abbildungslegende nichts anderes ergibt. Sofern das betreffende Material nicht unter der genannten Creative Commons Lizenz steht und die betreffende Handlung nicht nach gesetzlichen Vorschriften erlaubt ist, ist für die oben aufgeführten Weiterverwendungen des Materials die Einwilligung des jeweiligen Rechteinhabers einzuholen.

Weitere Details zur Lizenz entnehmen Sie bitte der Lizenzinformation auf http://creativecommons.org/ licenses/by/4.0/deed.de.

Interessenskonflikt Es gibt keinerlei Interessenskonflikte im Zusammenhang mit der Abfassung dieses Beitrags.

\section{Literatur}

Autor, D., Dorn, D., Katz, L. F., Patterson, C., \& Van Reenen, J. (2017). Concentrating on the fall of the labor share. American Economic Review, 107(5), 180-185.

Autor, D. H., Dorn, D., \& Hanson, G. H. (2013). The China syndrome: local labor market effects of import competition in the United States. American Economic Review, 103(6), 2121-2168.

Bagwell, K., \& Staiger, R. W. (1999). An economic theory of GATT. American Economic Review, 89(1), 215-248.

Bagwell, K., \& Staiger, R. W. (2000). GATT-Think. Bd. 8005. Cambridge/Ma.: National Bureau of Economic Research.

Baldwin, R.E. (1989). The political economy of trade policy. Journal of Economic Perspectives, 3(4), $119-135$.

van Bergeijk, P. A. G. (2018). On the brink of deglobalisation . . . again. Cambridge Journal of Regions, Economy and Society, 11(1), 59-72.

van Bergeijk, P. A. G. (2019). Deglobalization 2.0: trade and openness during the great depression and the great recession. Cheltenham: Edward Elgar.

Bhagwati, J.N., \& Ramaswami, V.K. (1992). Domestic distortions, tariffs and the theory of optimum subsidy. Development economics, 3, 193-199.

Bordo, M.D. (2017). The second era of globalization is not yet over: an historical perspective. NBER Working Paper Series, Bd. 23786.

Cernat, L., \& Mustilli, F. (2018). Trade and labour market adjustments: what role for the European globalisation adjustment fund? Intereconomics, 53(2), 79-86.

Dauth, W., Findeisen, S., \& Südekum, J. (2017). Trade and manufacturing jobs in Germany. American Economic Review, 107(5), 337-342.

Kindleberger, C.P. (1973). The world in depression. Berkeley: University of California Press.

Krueger, A.O. (1996). The political economy of trade protection. Cambridge/Ma.: National Bureau of Economic Research.

Krugman, P. R. (1987). Is free trade Passé? Journal of Economic Perspectives, 1(2), 131-144.

Krugman, P.R. (1991). The move toward free trade zones. Policy implications of trade and currency zones, a symposium Spon- sored by the federal reserve bank of Kansas City, Jackson Hole, Wyoming. https://www.kansascityfed.org/publicat/sympos/1991/S91krugm.pdf. Zugegriffen: 17.8.2020. 
Krugman, P.R., Obstfeld, M., \& Melitz, M.J. (2018). International economics: theory and policy (11. Aufl.). London: Pearson.

Minford, P. (2017). From project fear to project prosperity. An introduction. Economists for free trade. https://www.economistsforfreetrade.com/wp-content/uploads/2017/08/From-Project-Fearto-Project-Prosperity-An-Introduction-15-Aug-17-2.pdf. Zugegriffen: 20.8.2020.

Olson, M. (1965). The logic of collective action: public goods and the theory of groups. Cambridge: Harvard University Press.

van Paridon, K. (2018). Towards a more inclusive future: on the delicate balance between economic change and social stability in a time of globalisation. List Forum für Wirtschafts- und Finanzpolitik, 44(2), 241-281.

Rodrik, D. (1995). Political economy of trade policy. In Handbook of international economics (Bd. 3, S. 1457-1494). Columbia: CEPR, NBER.

Sachverständigenrat (2018). Vor wichtigen wirtschaftspolitischen Weichenstellungen. Jahresgutachten 2018/19. Wiesbaden: Sachverständigenrat zur Begutachtung der gesamtwirtschaftlichen Entwicklung.

Südekum, J. (2017). Die Globalisierungsverlierer kompensieren - Aber wie? Wirtschaftsdienst, 97(8), 566-570.

Tucholsky, K. (1931). Kurzer Abriss der Nationalökonomie. https://tucholsky.de/kurzer-abriss-dernationaloekonomie/. Zugegriffen: 20.8.2020.

World Trade Organization (2019). Annual Report 2019.

World Trade Organization (2020). Goods barometer confirms steep drop in trade but hints at nascent recovery. https://www.wto.org/english/news_e/news20_e/wtoi_19aug20_e.htm. Zugegriffen: 19.8.2020. 